\title{
Cocaine Enhances HIV-1 Replication in CD4+ T Cells by Down-Regulating MiR-125b
}

\author{
Chinmay K. Mantri, Jui Pandhare Dash, Jyoti Velamarti Mantri, Chandravanu C. V. Dash*
}

The Laboratory of Retrovirology and Epigenetics, Center For AIDS Health Disparities Research, Vanderbilt-Meharry Center for AIDS Research, Department of Biochemistry and Cancer Biology, Meharry Medical College, Nashville, Tennessee, United States of America

\begin{abstract}
The main objective of this study was to examine effects of cocaine on HIV-1 replication in primary CD4+ T cells. Cocaine a commonly used drug among HIV-1 positive individuals serves as a cofactor for HIV-1 infection and progression to acquired immunodeficiency syndrome (AIDS). Accumulating evidence suggest that cocaine increases HIV-1 replication in cell cultures, peripheral blood mononuclear cells (PBMCs) and animal models. Intriguingly, there are no studies on cocaineinduced alterations in HIV-1 replication in primary CD4+ T cells that serve as the main targets for HIV-1 replication in vivo. In this report, we demonstrate cocaine-induced enhancement of HIV-1 replication in primary CD4+ T cells isolated from human PBMCs. To decipher a potential mechanism, we examined whether cocaine targets the innate antiviral immunity of CD4+ T cells mediated by cellular microRNAs (miRNAs). This is because recently a network of anti-HIV miRNAs in CD4+ T cells is highlighted to suppress viral replication. Our genome wide miRNA expression analysis indicated downregulation of several anti-HIV miRNAs (miR-28, miR-125b, miR-150, miR-223, and miR-382) in cocaine treated CD4+ T cells. However, our real-time quantitative PCR analysis revealed significant downregulation of miR-125b only. Our results illustrated that miR-125b knockdown enhances HIV-1 replication, whereas overexpression of miR-125b decreases HIV-1 replication in these cells. Therefore, we believe miR-125b is a key player for the cocaine induced enhancement of HIV-1 replication in CD4+ T cells. Since, miR-125b targets the $3^{\prime}$ UTR regions of HIV-1 transcripts and inhibits viral protein translation, our data suggest modulation of post entry steps of HIV-1 by cocaine. Given that a plethora of studies suggest that cocaine regulates HIV entry, our results implicate a potentially novel mechanism by which cocaine can increase viral replication in CD4+ T cells.
\end{abstract}

Citation: Mantri CK, Pandhare Dash J, Mantri JV, Dash CCV (2012) Cocaine Enhances HIV-1 Replication in CD4+ T Cells by Down-Regulating MiR-125b. PLoS ONE 7(12): e51387. doi:10.1371/journal.pone.0051387

Editor: K.T. Jeang, National Institute of Health, United States of America

Received May 31, 2012; Accepted November 2, 2012; Published December 12, 2012

Copyright: (c) 2012 Mantri et al. This is an open-access article distributed under the terms of the Creative Commons Attribution License, which permits unrestricted use, distribution, and reproduction in any medium, provided the original author and source are credited.

Funding: This work is partly supported by grants (DA024558, DA30896, DA033892) from National Institute on Drug Abuse/National Institutes of Health, the Vanderbilt CTSA grant UL1 RR024975-01 from NCRR/NIH, and the Meharry Translational Research Center (MeTRC) CTSA grant (U54 RR026140 from NCRR/NIH and U54 MD007593 from NIMHD/NIH to CD. The funders had no role in study design, data collection and analysis, decision to publish, or preparation of the manuscript.

Competing Interests: The authors declare that no competing interests exist.

* E-mail: cdash@mmc.edu

\section{Introduction}

Illicit drug use remains the second most frequent mode of acquisition of HIV and drugs such as amphetamines, cocaine, marijuana, and opiates serve as cofactors for susceptibility to HIV infection and disease progression [1-8]. Cocaine, a commonly used drug among HIV-1 positive individuals, serves as a cofactor for HIV-1 infection and progression to acquired immunodeficiency syndrome (AIDS) [9-13]. Epidemiological studies suggest that HIV positive cocaine users have lower CD4+ T cell counts, increased risk of diseases progression and AIDS-related death [1113]. In vitro cocaine increases $\mathrm{HIV}-1$ replication in human peripheral blood mononuclear cells (PBMCs) and enhances viral load in humanized mice [14-16]. Although, cocaine is known to have immunomodulatory function [17-19], the underlying mechanism by which cocaine increases HIV-1 replication remains unclear. It has been proposed that cocaine increases HIV-1 infection/replication by inhibiting HIV-1 protective chemokines and/or upregulating the HIV-1 entry co-receptor [20-21]. For example, members of the $\beta$-chemokine family that bind to CCR5 such as regulated upon-activation $\mathrm{T}$ expressed and secreted (RANTES), macrophage inflammatory protein la (MIP-1a), and
MIP-1b have been demonstrated to inhibit entry of certain HIV-1 strains [22]. In addition, proteomics analysis of cocaine treated PBMCs isolated from HIV-positive donors suggests that cocaine differentially regulates expression of several key host proteins that may influence HIV-1 replication [23]. Since these studies were conducted using cell culture models or the mixed cell populations of PBMCs, there are no reports on primary CD4+ T cells. Given that CD4+ T cells are the main targets for HIV-1 infection and replication in vivo [24], it is critical to evaluate whether cocaine enhances HIV-1 replication in primary CD4+ T cells.

MicroRNAs (miRNAs) are noncoding single-stranded small RNAs that bind to the $3^{\prime}$ untranslated region (UTR) of target mRNA and post-transcriptionally regulate gene expression via degradation of specific mRNAs and/or repression of their translation [25]. Therefore, cellular miRNAs play critical roles for the normal function and diseased state of cells [25]. MiR-125 is a highly conserved miRNA from nematode to humans and has recently emerged as a key regulator of cell survival and differentiation [26]. In addition miR-125b has been shown to be dysregulated in multiple malignancies [26]. miR-125b is the ortholog of lin-4 in Caenorhaditis elegans and is transcribed from two loci located on chromosomes 11q23 (hsa-miR-125b-1) and 21q21 
(hsa-miR-125b-2) [27]. miR-125b has been reported to target many key proteins that regulate apoptosis, innate immunity, inflammation and hematopoietic differentiation [26]. Recently, miR-125b has been demonstrated to regulate a network of genes in CD4+ $\mathrm{T}$ cells that are critical for its differentiation [28]. Intriguingly, miR-125b also belongs to the network of cellular antiHIV miRNAs that suppress viral replication [29-30]. These antiHIV miRNAs target the 3' UTR regions of HIV-1 transcripts and inhibit HIV-1 replication. It has been proposed that miR-125b and other anti-HIV-1 miRNAs may be responsible for inducing latency in naïve CD4+ T cells [29]. Very recently, it has also been suggested that downregulation of miR-125b in the PBMCs of HIV-1 infected individuals may lead to viremia [31].

Since CD4+ T cells serve as primary targets for HIV-1 infection and replication [24], in this study we have examined whether cocaine enhances HIV-1 replication in CD4+ T cells. Using primary CD4+ T cells isolated from human PBMCs, we illustrate cocaine-induced increase in HIV-1 replication in these cells. In an attempt to decipher the mechanism by which cocaine enhances HIV-1 replication, we examined whether cocaine targets the antiHIV-1 miRNAs in CD4+ T cells. The rationale is derived from the accumulating evidence that cellular miRNAs confer antiviral innate immunity and may negatively regulate HIV-1 replication [29-30,32-33]. Therefore, we carried out genome wide miRNA expression analysis to investigate whether cocaine modulates cellular anti-HIV-1 miRNA expression in primary CD4+ T cells. Our genome wide miRNA results indicated downregulation of several anti-HIV-1 miRNAs. However, our real time PCR analysis demonstrated substantial downregulation of miR-125b in uninfected and infected activated CD4+ T cells. This cocaine induced downregulation of miR-125b resulted in increased HIV-1 replication in CD4+ T cells. This was confirmed by knock-down and overexpression studies of miR-125b. Furthermore, our promoter reporter assay revealed that cocaine treatment resulted in downregulation of miR-125b promoter activity. Given that miR-125b inhibits HIV-1 protein translation, the data presented in this report demonstrate a role of post entry steps of HIV-1 by which cocaine enhances HIV-1 replication. Therefore, our results implicate a potentially novel mechanism by which cocaine can increase viral replication in HIV- 1 positive drug addicts.

\section{Materials and Methods}

\section{Healthy Donors, Isolation of PBMCs, Purification of CD4+ T Cells and Cell Culture}

Human blood was purchased from the New York Blood Center as per the Meharry Medical College IRB from 12 healthy donors. For PBMC isolation fresh human blood was diluted 1:2 with Phosphate Buffered Saline (PBS). Subsequently, $25 \mathrm{ml}$ of diluted blood was overlaid on $12.5 \mathrm{ml}$ of Ficoll-Paque ${ }^{\mathrm{TM}}$ Premium reagent (GE) in a $50 \mathrm{ml}$ conical tube and centrifuged at $750 \times \mathrm{g}$ without break for 20 minutes at $20^{\circ} \mathrm{C}$. Thereafter, the interphase cells (PBMCs) were transferred carefully to a new $50 \mathrm{ml}$ tube and PBS was added to make up to $50 \mathrm{ml}$. Subsequently, the PBMCs were centrifuged several times and washed with PBS to remove unwanted cell types. The resulting cell pellet was resuspended in PBS followed by counting and viability determination by trypan blue exclusion. CD4+ T cells were isolated by negative selection as per the standard protocol described in $\mathrm{CD} 4^{+} \mathrm{T}$ cell Isolation Kit II (Miltenyi Biotec). The purity of isolated CD4+ T cells was checked by Flow Cytometry (see below). The CD4+ T cells were activated by PHA $(5 \mathrm{mg} / \mathrm{ml})$ for $48 \mathrm{~h}$, and maintained with interleukin-2 (20 U/ml; Sigma). SupT1, a T cell line, was obtained from American Type Culture Collection (ATCG) and maintained in complete RPMI (cRPMI) that contains RPMI with 10\% fetal bovine serum (FBS) and antibiotics. ACH-2 and TZM-bl cells were obtained from NIH AIDS Research and Reference Reagent Program, Division of AIDS, NIAID, NIH: ACH-2 cells from Dr. Thomas Folks [34-35] and TZM-bl cells from Dr. John G. Kappes, Dr. Xiaoyun Wu and Tranzyme [36].

\section{Cocaine Treatment}

Cocaine hydrochloride was obtained from the National Institute on Drug Abuse (NIDA) Drug Supply Program. We use $1 \mu \mathrm{M}$ cocaine in our studies because of its relevance to human plasma levels of drug users that often range between $0.4-1.6 \mu \mathrm{M}$ for intranasal administration [37-38].

\section{Microarray Analysis}

Purified CD4+ T cells were treated with cocaine for 24 hours and total RNA was isolated with miRNeasy mini Kit (Qiagen). RNA processing, microarray fabrication, hybridization, data acquisition and analysis were performed at the Vanderbilt University Genomics Core. Briefly, $1 \mu \mathrm{g}$ of total RNA (control and treated, in triplicates) was used for miRNA expression profiling using the miRGURY LNA microRNA Arrays version 11.0 (Exiqon, DK). Microarray data acquisition was done with GenePix Pro v. 6.1 acquisition \& Analysis Software. miRNA microarray results were analyzed using an ANOVA statistical test.

\section{Real Time RT-PCR Analysis}

HIV-1 infected and uninfected CD4+ T cells were treated with cocaine and total RNA was isolated by miRNeasy mini Kit (Qiagen). Real Time RT-PCR was carried out with the RNA using miRNA specific primers (Exiqon) in C1000 Touch $^{\mathrm{TM}}$ Thermal cycler (Bio-Rad). PCR results were analysed in Bio-Rad CFX manager software. miRNA expression levels were normalized to 5s-rRNA and GAPDH expression.

\section{Infectious and Pseudotyped Virus Production}

For infectious HIV-1, we use the supernatant of chronically infected ACH-2 cells (with HIV-1 LAI isolate). ACH-2 cells were plated $\left(5 \times 10^{5}\right.$ cells $\left./ \mathrm{ml}\right)$ in cRPMI media overnight and were activated with PMA and TNF- $\alpha$ for $1 \mathrm{hr}$. Thereafter, the cells were washed twice with PBS and incubated overnight at $37^{\circ} \mathrm{C}$ with fresh cRPMI media. The virus containing supernatant was collected by centrifugation and filtering through a $0.45 \mu \mathrm{M}$ filter. We generated VSV-G pseudotyped HIV-1 by transfecting the pNL4.3 molecular clone of HIV-1 and pVSV-G into 293T cells using Lipofectamine (Invitrogen). After $48 \mathrm{hr}$, culture supernatant containing the VSV-G pseudotyped virions was collected, centrifuged and filtered through a $0.45 \mu \mathrm{M}$ membrane. pLVSV$\mathrm{G}$ and pNL4.3dE.dV-Luc (a gift of Dr. Vineet KewalRamani, NCI/NIH) and pNL4.3dE.dV-RFP (NIH AIDS Reagent Program) were transfected to $293 \mathrm{~T}$ cells and the virus was collected after 48-72 hr. Concentration of the virus was determined by p24 ELISA assay and infectivity was measured by luciferase reporter assay using TZM-bl cells which harbors a firefly luciferase reporter gene under the control of HIV-1 promoter.

\section{Infection}

PHA Activated CD4+ T cells $\left(1 \times 10^{6}\right.$ cells $)$ were infected with HIV-1 LAI ( MOI of 5 ) or VSV-G pseudotyped virus (MOI 0.1 ) by spinoculation in the presence of polybrene (Sigma) and were cultured $\left(4 \times 10^{5} \mathrm{cells} / \mathrm{ml}\right)$ for $1-2$ weeks in the presence or absence of cocaine. Productive infection was measured by FACS by detecting intracellular HIV-1 p24 protein. TZM-bl cells that 
express CD4 and CXCR4/CGR5 receptors and harbor an integrated copy of firefly luciferase gene under the control of HIV-1 LTR promoter, were infected in the presence of polybrene (Sigma) for 6 hours. Thereafter, cells were washed with PBS and were incubated at $37^{\circ} \mathrm{C}$. After $48-72 \mathrm{hrs}$, TZM-bl cells were washed, lysed and luciferase activity was measured using a Synergy HT Multi-Mode Microplate Reader.

\section{Knockdown and Overexpression of miR-125b in CD4+ T Cells}

miR-125b inhibitors and negative controls were purchased from Dharmacon (Lafayette, CO). 10-100 pmole of anti-miRNAs or negative control was transfected to SupT1 cells using Neon Transfection System (Invitrogen). Cells were recovered in prewarmed antibiotic-free RPMI medium and incubated for $3 \mathrm{~h}$ at $37^{\circ} \mathrm{C} / 5 \% \mathrm{CO}_{2}$. Thereafter, these cells were infected to determine the effect of miR-125b on HIV-1 replication.

miR-125b mimic and negative controls were purchased from Dharmacon (Lafayette, CO). 100-200 pmole of miR-125b mimic or negative control was transfected to CEM cells (a gift from Richard D'Aquila, Vanderbilt) using Neon Transfection System (Invitrogen). Cells were recovered in pre-warmed antibiotic-free RPMI medium and incubated for $3 \mathrm{~h}$ at $37^{\circ} \mathrm{C} / 5 \% \mathrm{CO}_{2}$. Thereafter, these cells were infected to determine the effect of miR-125b on HIV-1 replication.

\section{miR-125b Complementation Assay}

SupT1 cells $\left(5 \times 10^{4}\right)$ were infected with VSV-G pseudotyped HIV (MOI $\sim 0.1$ ) by spinoculation in the presence of polybrene and the infected cells were grown in presence of cocaine for $36 \mathrm{hr}$. Thereafter, cells were transfected with 100 pmole of miR-125b mimic or negative control using Neon Transfection System (Invitrogen) and cells were recovered in pre-warmed antibioticfree RPMI medium. After $2 \mathrm{hr}$ incubation cells were transferred to complete RPMI medium and cultured for another $24 \mathrm{hr}$ at $37^{\circ} \mathrm{C} /$ $5 \% \mathrm{CO}_{2}$. Thereafter, these cells were harvested for Luciferase assay by Luciferase Assay System (Promega). Luciferase activity was measured using Synergy HT Multi-Mode Microplate Reader (BioTek) and normalized to total protein content of the lysate.

\section{miR-125b Promoter Transcription Assay}

pGL3 Basic (Promega) and pGL3-miR-125b-1 reporter (a kind gift from Dr. Xian Ming Chen, Creighton University Medical Center, Omaha, NE ) containing miR-125b promoter was transfected into 293T cells using Lipofectamine (Invitrogen) for $24 \mathrm{hr}$. Luciferase assay was carried out after treating the cells with $0.1 \mu \mathrm{M}$ and $1 \mu \mathrm{M}$ cocaine for $4 \mathrm{hr}$. Luciferase activity was measured using Synergy HT Multi-Mode Microplate Reader (BioTek) and normalized to total protein content of the lysate. The luciferase activity of miR-125b construct was compared with that of the promoter less pGL3 basic vector.

\section{FACS Analysis}

To check the purity of isolated CD4+ T cells, cells were washed twice with PBS containing $0.25 \%$ EDTA and $0.5 \%$ BSA and incubated with anti-CD4 antibodies for 15 minute at $4^{\circ} \mathrm{C}$ in the same buffer. After staining cells were washed two times with the same buffer. For intracellular p24 staining, cells were first fixed with $4 \%$ formaldehyde solution and permeabilised for 20 minutes in BD Cytofix/Cytoperm ${ }^{\text {TM }}$ Plus Permeabilization Buffer. After permeabilization, cells were incubated with $5 \mu \mathrm{l}$ of anti-p24 antibody or IgG isotype control antibody for $20 \mathrm{~min}$ at $4{ }^{\circ} \mathrm{C}$. Then cells were washed twice with BD Cytofix/Cytoperm ${ }^{\mathrm{TM}}$ Plus
Permeabilization Buffer and resuspended in the same buffer. Cells were analyzed on a BD FACSCalibur ${ }^{\mathrm{TM}}$ platform and data analysis was done with Cell Quest Pro (BD) or FlowJo (Tristar) software. FITC-anti-CD4; FITC- and PE- anti-CD25; and FITCIgG antibodies were obtained from Miltenyi Biotec. Anti-p24FITC antibody was obtained from Beckman Coulter.

\section{Results}

\section{Cocaine Enhances HIV-1 Replication in Primary CD4+ T Cells}

It has been described that cocaine enhances HIV-1 replication in PBMCs and animal models [14-16]. Surprising, there are no reports on the effects of cocaine on HIV-1 replication in CD4+ T cells that are the primary targets of HIV-1. To test this, we isolated PBMCs from peripheral blood of normal human donors and purified CD4+ T cells by negative selection. These primary CD4+ $\mathrm{T}$ cells were activated and infected with infectious HIV-1 and treated with cocaine after infection. Productive infection was examined after 7 days by detecting intracellular viral p24 antigen by FACS. Our FACS analysis illustrate that cocaine enhances HIV-1 replication in primary $\mathrm{CD} 4+\mathrm{T}$ cells (Fig. 1), since significantly higher percentage of cells expressed viral p24 in the presence of cocaine in comparison to in the untreated cells. Importantly, this enhancement is not dependent on donors used as reflected in the data presented in Fig. 1C that illustrate a consistent increase in HIV-1 replication in the presence of cocaine. To our knowledge these data sets provide the first evidence on cocaineinduced enhancement of HIV-1 replication in primary CD4+ T cells.

\section{Cocaine Enhances HIV-1 Replication by Modulating Post Entry Steps}

HIV-1 entry is mediated by CD4 receptor and CXCR4/CCR5 co-receptors [39-41]. It has been proposed that cocaine increases HIV-1 replication by either upregulating these entry co-receptor or inhibiting HIV-1 suppressing chemokines on the target cell [2021]. In our experiment described in Fig. 1 however, cocaine was added to the cells post-infection. Therefore we hypothesized that cocaine may modulate viral post entry steps. To examine this, we abrogated entry receptor (CD4 and CXCR4/CCR5) requirement by pseudotyping HIV-1 virions with the vesicular stomatitis virus glycoprotein (VSV-G). VSV-G pseudotyped virions are known to mediate viral entry through endocytosis [42]. These pseudotyped virions were used to infect TZM-bl cells that harbor an integrated copy of firefly luciferase gene under the control of HIV-1 LTR promoter. After 48-72 hrs, the cells were lysed and luciferase activity was measured. Results from this experiment demonstrate increased HIV-1 transcription as measured by luciferase activity in the presence of cocaine (Fig. 2). Since pseudotyped HIV-1 does not depend on CD4 and CXCR4/CCR5 for entry, our data in Fig. 2 strongly suggest that cocaine modulates viral post entry steps to enhance HIV-1 replication. Therefore, we propose that cocaine impacts both the viral entry and post entry steps to enhance HIV-1 replication.

\section{Cocaine Down-regulates miR-125b in CD4+ T Cells}

In an attempt of decipher the specific steps of viral post entry that is targeted by cocaine, we examined whether cocaine modulates the anti-HIV-1 miRNAs in CD4+ $\mathrm{T}$ cells. This is because a family of anti-HIV cellular miRNAs has been recently described in CD4+ $\mathrm{T}$ cells that confer antiviral innate immunity [29]. Therefore, we carried out genome wide miRNA analysis to investigate whether cocaine modulates cellular anti-HIV miRNA 
(A) Donor 1

I

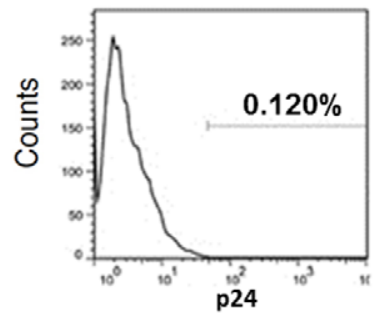

II

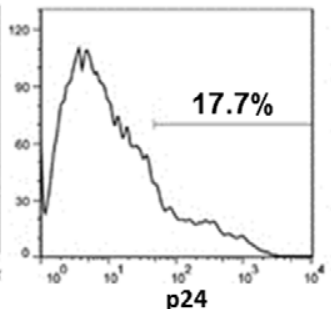

III

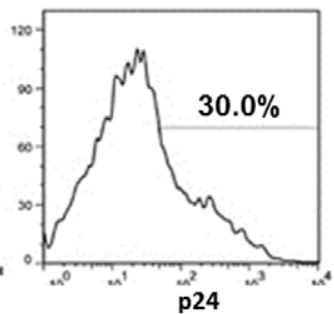

(C)

(B) Donor 2

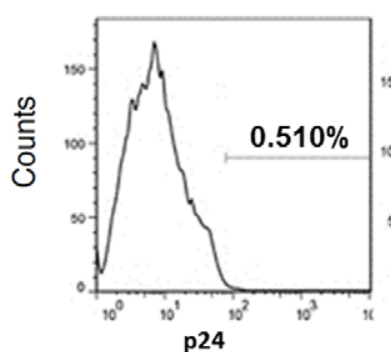

II

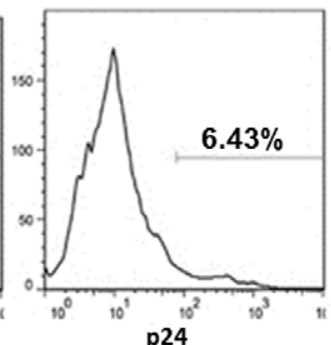

III

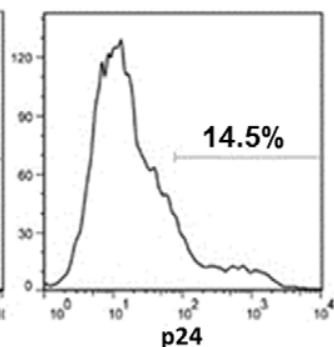

Donor Fold increase in infection

1

2

1.69

2.25

1.43

1.51

1.64

1.56

1.37

1.35

1.43

1.37

Figure 1. Cocaine-enhances HIV-1 replication in primary CD4+ T cells. Primary CD4+ T cells were isolated by negative selection from human PBMCs. After isolation, these cells were activated by PHA for 48-72 hrs and infected with infectious HIV- 1 LAl by spinoculation and cultured in the presence or absence of cocaine. Productive infection was measured by detecting viral p24 protein 7 days post infection by FACS. (A-B) Representative data from two donors. Panels I: p24 staining of uninfected cells. Panels II: p24 staining of HIV-1 infected cells in the absence cocaine. Panels III: p24 staining of HIV-1 infected cells in the presence of cocaine. (C) Data from ten donors with fold increase in HIV-1 replication in presence of cocaine. Cocaine treatment significantly increased the percentage of cells expressing viral p24 protein in these cells. Data are representative of three independent experiments comprising nine different donors. doi:10.1371/journal.pone.0051387.g001

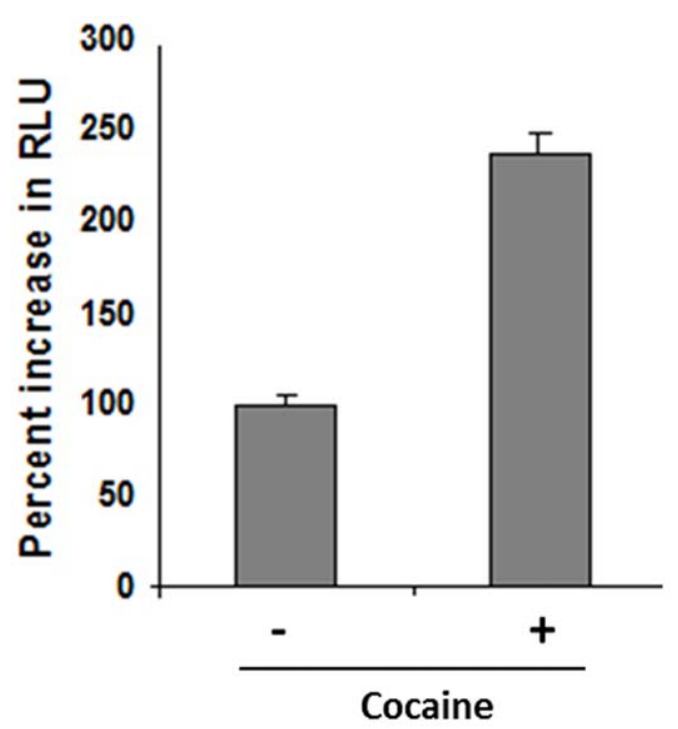

Figure 2. Cocaine-enhances HIV-1 replication by targeting post entry steps. To examine whether cocaine targets post entry steps of HIV-1 life cycle, we abrogated cellular receptor requirement for viral entry by pseudytyping HIV-1 virions with VSV-G envelop. Thereafter, TZM-bl cells (that harbor an integrated copy of luciferase reporter gene under the control of HIV-1 LTR promoter) were infected with VSV-G pseudotyped HIV-1 virions in the presence and absence of cocaine. Productive infection was measured after $48-72$ hrs by HIV- 1 LTR driven luciferase expression. Data are representative of three independent experiments conducted in triplicates. doi:10.1371/journal.pone.0051387.g002 expression in primary $\mathrm{CD} 4+\mathrm{T}$ cells. Our microarray data revealed downregulation of an array of cellular miRNAs (data not shown) including anti-HIV-1 miRNAs (miR-125b, miR-150, miR-28-5p, miR-223, and miR-382) in cocaine treated cells (Fig. 3A). It has been previously described that these anti-HIV-1 miRNAs target the $3^{\prime}$ UTR regions of HIV-1 transcripts and inhibit viral translation [29-30]. Therefore, we carried out real time PCR analysis to confirm our microarray data and the results are presented in Fig. 3B. Our data illustrate that cocaine treatment significantly downregulated "miR-125b" expression in these cells. Other anti-HIV-1 miRNAs that were downregulated in microarray analysis (Fig. 3A) were not downregulated in our real time PCR experiments. Quantitative real-time PCR (qPCR) is commonly used to validate gene expression results obtained from microarray analysis. However, microarray and qPGR data often result in disagreement and both techniques suffer from pitfalls [43]. For example, the quality of gene expression data obtained from microarrays can vary greatly with platform and procedures used [43]. Therefore, the pairing of microarray and qPCR is common in gene expression studies that produce more reliable results [43]. Given that both real time PCR and microarray analysis demonstrated downregulation of miR-125b, we believe the effects of cocaine on miR-125b are real. Therefore, we tested a role of miR-125b in HIV-1 replication.

\section{Cocaine-induced Down-regulation of miR-125b Enhances HIV-1 Replication in CD4+ T Cells}

Since miR-125b was significantly downregulated in cocaine treated CD4+ T cells both by microarray and real time PCR analysis, we evaluated whether downregulation of miR-125b can enhance HIV-1 replication. To achieve this we used the T cell line 
(A)

\begin{tabular}{|c|c|c|}
\hline Anti-HIV miRNA & $\begin{array}{c}\text { Fold } \\
\text { Change }\end{array}$ & p-value \\
\hline hsa-miR-125b & -1.86704 & 0.001999 \\
\hline hsa-miR-150* & -1.90992 & 0.000248 \\
\hline hsa-miR-28-5p & -1.9244 & 0.000731 \\
\hline hsa-miR-223 & -1.83294 & 0.000765 \\
\hline hsa-miR-382 & -1.88392 & 0.010829 \\
\hline
\end{tabular}

(B)

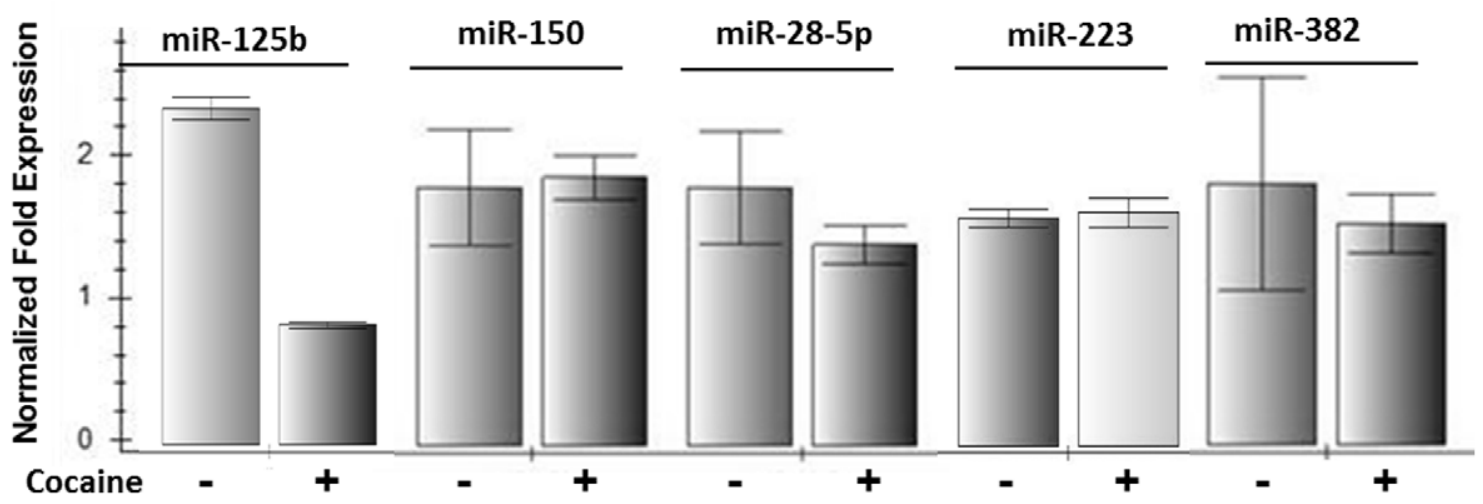

Figure 3. Cocaine downregulates anti-HIV-1 miRNAs in CD4+ T cells. Activated primary CD4+ T cells were treated with cocaine for 24 hrs and expression of cellular miRNAs were analyzed by microarray and compared with untreated cells. The expressions of cellular anti-HIV-1 miRNAs with highly significant $p$ values were considered. The table shows that cocaine treatment downregulated a family of anti-HIV-1 miRNAs including miR125b, miR-150, miR-28p, miR-223, and miR-382 in CD4+ T cells. (B) To confirm our microarray data, we carried out real time PCR analysis using RNA isolated from cocaine treated CD4+ T cells and compared expression of these miRNAs with untreated cells. miRNA expression levels were determined by miRNA specific primers and normalized to 5s-rRNA and GAPDH expression. miR-125b was consistently downregulated upon cocaine treatment, whereas expression of other miRNAs (miR-26, miR-150, miR-223, miR-122, and miR-296) were not significantly affected. Data are representative of three independent experiments comprising six to nine different donors. doi:10.1371/journal.pone.0051387.g003

SupT1 since it supports HIV-1 replication. Most importantly, SupT1 cells express miR-125b and cocaine treatment induces substantial downregulation of miR-125b expression (Fig. 4A). Therefore, we tested whether cocaine treatment can increase HIV-1 replication in SupT1 cells. We infected SupT1 cells with pseudotyped HIV-1 with a RFP reporter and analyzed productive infection by measuring RFP expression by FACS. Our results indicated that the percentage of cells expressing RFP is increased significantly when the cells were treated with cocaine (Fig. 4 C-D). Since this a single cycle replication assay, these results reaffirm that cocaine targets post entry steps of the viral life cycle to enhance HIV-1 replication.

\section{Knock-down of miR-125b Enhances HIV-1 Replication}

To evaluate whether cocaine targets miR-125b to enhance HIV-1 replication, we conducted knockdown experiments using anti-miR-125b and SupT1 cells. As illustrated in Fig. 5A, our knockdown experiments were designed to achieve the level of miR-125b expression to $50 \%$ that mimics cocaine-induced downregulation as presented in Fig. 3B and Fig. 4A. Subsequently, we infected these cells with VSV-G pseudotyped HIVl-RFP reporter virus and determined infection by measuring RFP expression by FACS after $48-72 \mathrm{hrs}$. A comparative analysis of infection experiments illustrates that knock-down of miR-125b enhanced RFP expression in SupT1 cells from 15\% to $41 \%$. This enhancement demonstrates that miR-125b plays a critical role for cocaine-induced increase of HIV-1 replication in CD4+ T cells.

Over-expression of miR-125b Inhibits HIV-1 Replication

To further validate a role of miR-125b in HIV-1 replication, we over-expressed miR-125b in CEM cells using miR-125b mimics. The rational for using CEM cells is that they express miR-125b at very low or undetectable levels and higher miR$125 \mathrm{~b}$ levels are achieved by transfection of miR-125b mimic (Fig. 6A). Infection of these cells with VSV-G pseudotyped HIV-1 RFP resulted in reduction of RFP expression in comparison to control cells (Fig. 6B-E). These data sets further support our arguments for a role of miR-125b in inhibiting HIV-1 replication (Fig. 6B-E).

\section{Effect of Cocaine on HIV-1 Replication is Dependent on miR-125b Expression}

To evaluate whether cocaine-induced downregulation of miR$125 \mathrm{~b}$ directly contributes to increased HIV-1 replication, we carried out complementation assay using miR-125b mimic. 
(A)

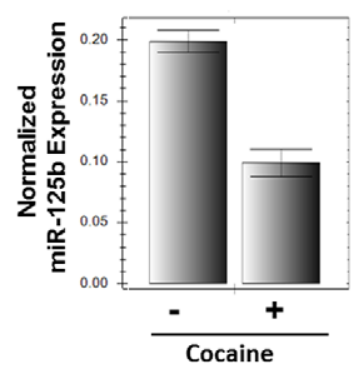

(B)

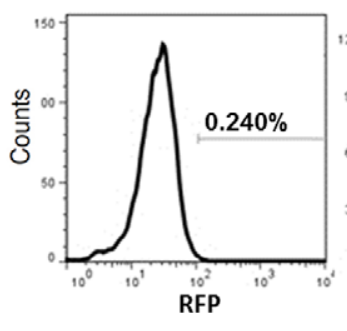

(C)

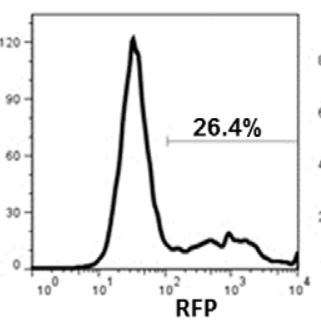

(D)

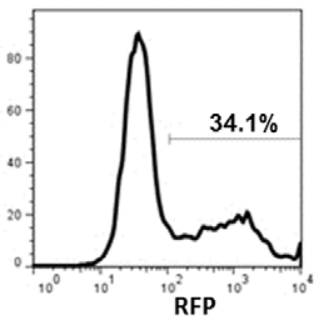

(E)

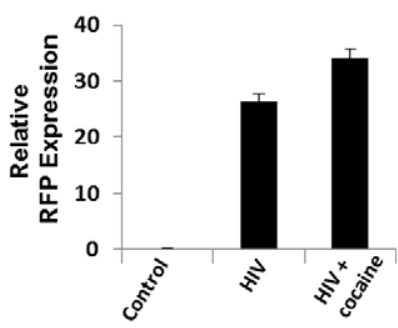

Figure 4. Cocaine induced downregulation of miR-125b enhances HIV-1 replication. We carried out single cycle replication assay to evaluate modulation of HIV-1 replication by cocaine. For this experiment we used a T cell line SupT1 that expresses miR-125b. (A) Cocaine treatment substantially downregulated miR-125b in SupT1 cells as determined by real time PCR. Pseudotyped HIV-1 virions with RFP reporter were used to infect SupT1 cells and HIV-1 replication was measured by detecting RFP expression by FACS 48-72 hr post infection. (B) RFP expression of uninfected cells. HIV-1 replication was measured in the absence (C) and presence (D) of cocaine. (E) Relative RFP expression from three independent experiments. Cocaine treatment resulted in increased RFP expression implying enhanced HIV-1 replication. Data are representative of three independent experiments conducted in triplicates.

doi:10.1371/journal.pone.0051387.g004

SupT1 cells were infected with VSV-G pseudotyped HIV-1Luciferase reporter virus in the presence of cocaine. Thereafter, miR-125b mimic was transfected to these cells. As expected cocaine enhanced HIV-1 replication in these cells as reflected by increased luciferase activity (Fig. 7). This increase in HIV-1 replication by cocaine was abrogated by miR-125b mimic expression. These results demonstrate that miR-125b plays a direct role for cocaine-induced increase in HIV-1 replication.

\section{Cocaine Downregulates miR-125b in HIV-1 Infected CD4+ T Cells}

To examine whether cocaine has the ability to downregulate miR-125b in HIV-1 infected cells, we infected primary CD4+ T cells with HIV-1 LAI and treated them with cocaine. We carried out RT-PCR experiment using RNA from these cells and data in Fig. 8 illustrate that upon cocaine treatment, miR-125b expression is downregulated in the infected cells.

\section{Cocaine Modulates miR-125b Promoter Driven Transcription}

To understand a mechanism by which cocaine downregulates miR-125b, we tested whether cocaine modulates the promoter activity of miR-125b. To test this, we transfected $293 \mathrm{~T}$ cells with a
pGL3 construct containing the miR-125b promoter that drives transcription of luciferase reporter (Fig. 9A). Thereafter, these cells were treated with cocaine at $0.1 \mu \mathrm{M}$ and $1 \mu \mathrm{M}$ concentrations. Upon cocaine treatment transcription of luciferase in these cells was decreased as measured by luciferase activity (Fig. 9B). These data sets suggest that cocaine regulate miR-125b expression by suppressing miR-125b transcription.

\section{Discussion}

Cocaine serves as a cofactor for susceptibility to HIV infection [11-12] and AIDS progression [13]. In addition, epidemiological studies indicate that HIV positive cocaine users have lower CD4+ $\mathrm{T}$ cell counts and have a significant acceleration of decline of CD4+ T cells [9-13]. In vitro cocaine increases HIV-1 replication in cell culture models, peripheral blood mononuclear cells (PBMCs) [14-15] and enhances viral load in humanized mouse models [16]. Since CD4+ T cells are the primary targets for HIV1 infection/replication in vivo [24], the goal of this study was to evaluate cocaine-induced enhancement of HIV-1 replication in primary CD4+ T cells [18]. Results presented here demonstrate for the first time that cocaine enhances HIV-1 replication in primary CD4+ T cells by downregulating the cellular anti-HIV



(B)

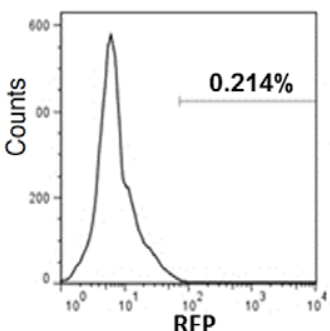

(C)

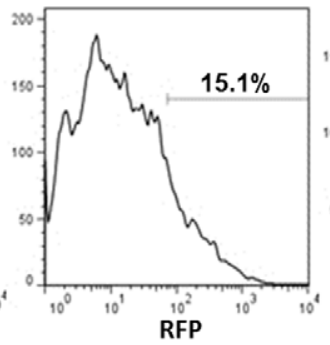

(D)

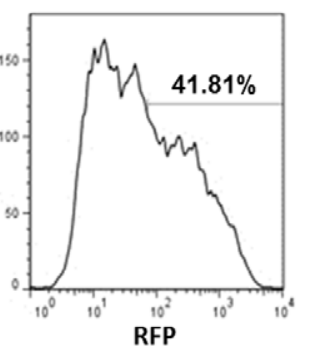

(E)

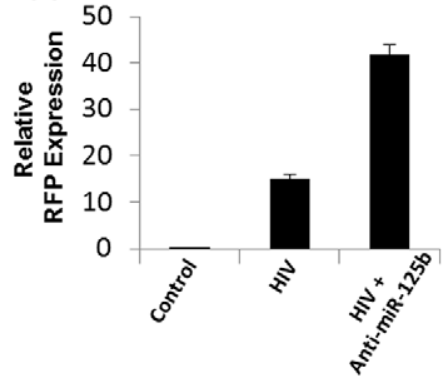

Figure 5. Knock-down of miR-125b enhances HIV-1 replication. (A) miR-125b knockdown experiments were conducted using anti-miR-125b and SupT1 cells. These cells were infected with VSV-G pseudotyped HIV-1-RFP reporter virus and infection was determined by FACS. Since cycle replication was determined by measuring intracellular RFP expression. (B) RFP expression of uninfected cells by FACS analysis. HIV-1 replication was measured in the absence $(\mathbf{C})$ and presence $(\mathbf{D})$ of anti-miR-125b. (E) Relative RFP expression from three independent experiments. miR-125b knockdown resulted in increased RFP expression implying miR-125b plays a critical role for cocaine-induced enhancement of HIV-1 replication. Data are representative of three independent experiments conducted in triplicates.

doi:10.1371/journal.pone.0051387.g005 
(A)

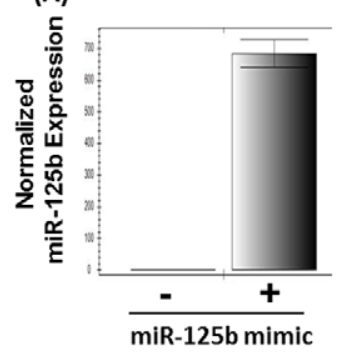

(B)

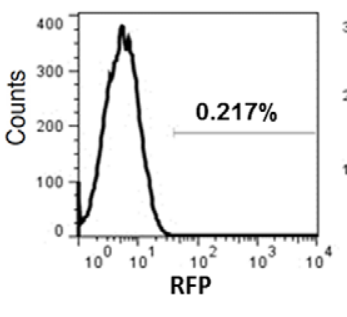

(C)

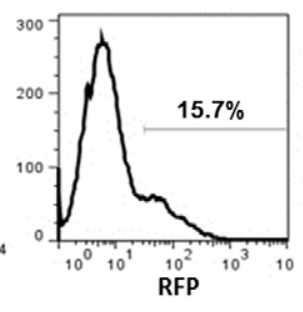

(D)



(E)

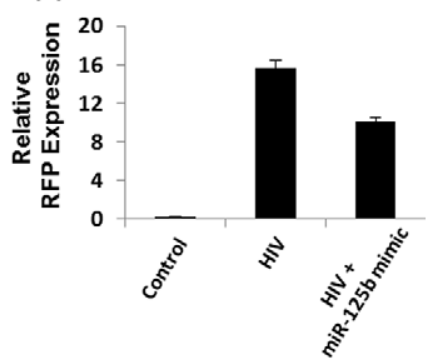

Figure 6. Over-expression of miR-125b inhibits HIV-1 replication. (A) miR-125b over-expression experiments were conducted using antimiR-125b and CEM cells. These cells were infected with VSV-G pseudotyped HIV-1-RFP reporter virus and infection was determined by FACS. Since cycle replication was determined by measuring intracellular RFP expression. (B) RFP expression of uninfected cells. HIV-1 replication was measured in the absence (C) and presence (D) of miR-125b mimic. (E) Relative RFP expression from three independent experiments. miR-125b over-expression resulted in decreased RFP expression implying miR-125b modulates HIV-1 replication. Data are representative of three independent experiments conducted in triplicates.

doi:10.1371/journal.pone.0051387.g006

miRNA miR-125b. These findings warrant a comprehensive understanding of cocaine-induced alterations in CD4+ $\mathrm{T}$ cell biology that will help us decipher the underlying mechanisms by which HIV-1 positive cocaine users have accelerated HIV-1 pathogenesis and AIDS-related death.

Although the mechanism by which cocaine enhances HIV replication remains unclear, there is strong evidence that cocaine modulates HIV-1 entry to the target cells [20-21]. HIV-1 entry is mediated by the binding of viral glycoproteins (HIV-1 gp120 and gp41) to CD4 and chemokine receptors (CGR5 and CXCR4) that leads to membrane fusion and release of viral core into the cytoplasm of target cells [36-38]. It has been proposed that cocaine enhances viral entry by inhibiting expression of HIVsuppressing chemokines in target cells [20-21]. These include regulated upon-activation $\mathrm{T}$ cell expressed and secreted (RANTES), macrophage inflammatory protein la (MIP-1a), and MIP-1b [22]. These molecules are known to inhibit binding/

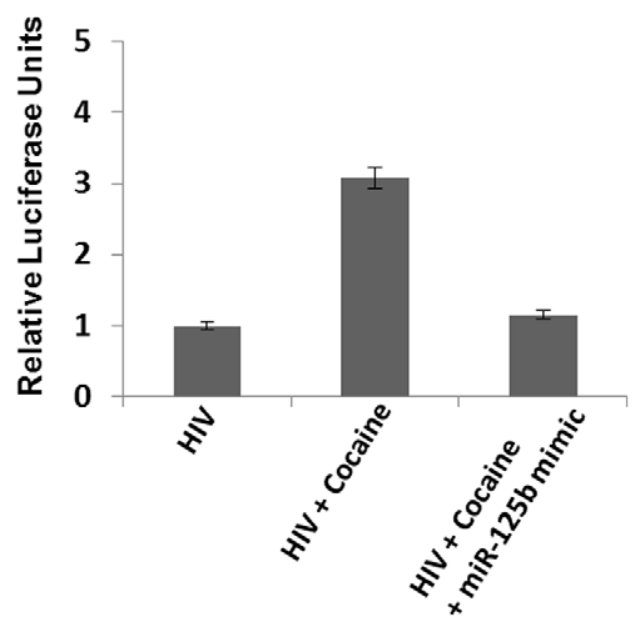

Figure 7. Effect of Cocaine on HIV-1 replication is dependent on miR-125b expression. SupT1 cells were infected with VSV-G pseudotyped HIV-1-Luciferase reporter virus and infection was determined by luciferase activity. For miR-125b complementation assay cocaine treated infected cells were transfected with miR-125b mimic. The increase in HIV-1 replication by cocaine was abrogated by miR$125 \mathrm{~b}$ mimic expression. Data are representative of three independent experiments conducted in triplicates.

doi:10.1371/journal.pone.0051387.g007 fusion/entry of the virus mediated by the HIV-1 envelope [22]. In addition, it has also been reported that cocaine enhances HIV-1 entry by upregulating the expression of entry co-receptors in the target cell [20-21]. These findings have been instrumental in depicting viral entry as the major target for cocaine induced enhancement of HIV-1 replication. In contrast to this accepted model, results presented in this study indicate that cocaine targets the post entry steps of HIV-1 life cycle since cocaine was added to the CD4+ T cells after infection (Fig. 1). This argument is further strengthened by the results that demonstrate cocaine-induced increased replication of VSV-G pseudotyped HIV-1 virions (Fig. 2). This is because pseudotyping allows HIV-1 to enter the target cell via endocytosis by abrogating CD4 and CCR5/ CXCR4 receptor requirement for virus entry. It is important to point out that the post entry events of HIV-1 replication can be broadly categorized into reverse transcription, integration, transcription, translation, assembly and release steps [24]. Our microarray and real time PCR analysis demonstrated that cocaine downregulated the cellular anti-HIV miRNA miR-125b in primary CD4+ $\mathrm{T}$ cells (Fig. 3). Given that miR-125b has been



Figure 8. Cocaine downregulates miR-125b in HIV-1 infected CD4+ $\mathbf{T}$ cells. Activated primary CD4+ T cells were infected with HIV-1 $\mathrm{LAl}$ and treated with cocaine. Cocaine treatment downregulated miR$125 \mathrm{~b}$ in infected primary CD4+ T cells as determined by real time PCR. Data are representative of three independent experiments conducted in triplicates.

doi:10.1371/journal.pone.0051387.g008 
(A)
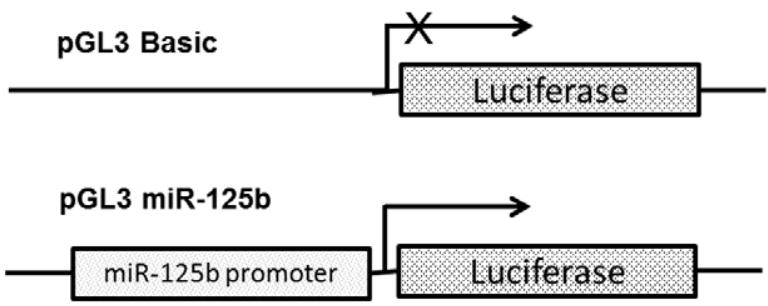

(B)

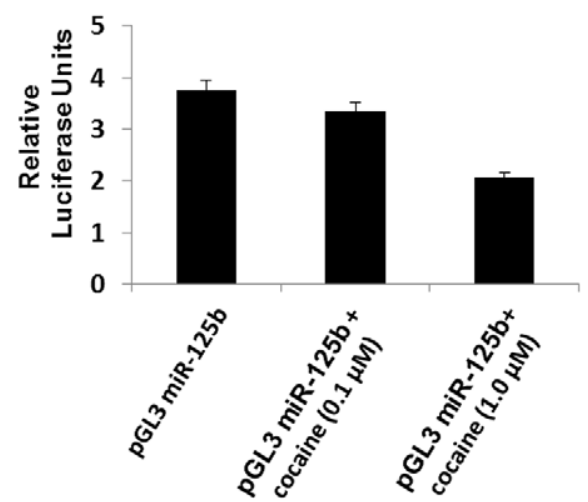

Figure 9. Cocaine regulates transcription of miR-125b. (A) A schematic representation of miR-125b promoter construct used. In this construct the miR-125b promoter drives the luciferase gene. (B) A construct containing miR-125b promoter driven luciferase gene or the vector control were transfected into 293T cells by Lipofectamine transfection. After $24 \mathrm{hrs}$, these cells were treated with cocaine $(0.1 \mu \mathrm{M}$ and $1 \mu \mathrm{M}$ ) for $4 \mathrm{hrs}$. Thereafter, cells were lysed and luciferase activity was measured by a luminometer. Cocaine treatment decreased miR125b driven luciferase expression. Data are representative of three independent experiments conducted in triplicates. doi:10.1371/journal.pone.0051387.g009

reported to inhibit viral translation, these data corroborate our contention that cocaine targets post entry steps of HIV life cycle. In addition, our data illustrated that cocaine down-regulates miR125b in HIV-1 infected CD4+ T cells.

miR-125b is a member of anti-HIV-1 miRNA family (including miR-28, miR-125b, miR-150, miR-223, and miR-382) that targets the 3 'UTR of HIV-1 transcripts and inhibit viral translation, a post entry step. Our contention is also supported by the recent report that morphine downregulates the expression of the antiHIV-1 miRNA in cultured human monocytes to enhance HIV-1 replication [44]. Therefore, based on these findings and available data, we propose that cocaine modulates both the entry and postentry steps in the target cells to enhance HIV-1 replication. We believe cocaine enhances $\mathrm{HIV}-1$ replication by targeting viral protein translation step in $\mathrm{CD} 4+\mathrm{T}$ cells, since knock-down of miR-125b resulted in increased HIV-1 expression and overexpression of miR-125b inhibited HIV-1 expression (Fig. 5-6).

\section{References}

1. Goedert JJ (1984) Recreational drugs: relationship to AIDS. Ann N Y Acad Sci. 437: $192-199$

2. Siegel L (1986) AIDS: relationship to alcohol and other drugs. J Subst Abuse Treat, 3: 271-274.

3. Donahoe RM, Falek A (1988) Neuroimmunomodulation by opiates and other drugs of abuse: relationship to HIV infection and AIDS. Adv Biochem Psychopharmacol 44: 145-158.

4. Friedman H (1996) Drugs of abuse as possible co-factors in AIDS progression: summary of panel discussion. Adv Exp Med Biol 402: 225-228.

5. Cabral GA (2006) Drugs of Abuse, Immune Modulation, and AIDS. J Neuroimmune Pharmacol 1: 280-295.
Although a body of recent literature suggests a multitude of regulatory functions of miR-125b in cell survival, differentiation and multiple malignancies (reviewed in [27]), the cellular targets of miR-125b in CD4+ T cells are not fully identified. miR-125b has been suggested to play a key role in maintaining the resting state of naive CD4+ $\mathrm{T}$ cells since it has been proposed to regulate a network of genes in CD4+ $\mathrm{T}$ cells that are critical for its differentiation [28]. It has also been suggested that miR-125b and other anti-HIV-1 miRNAs may be responsible for inducing latency in naïve CD4+ T cells by inhibiting viral translation [29]. Very recently, it has been reported that miR-125b is downregulated in the PBMCs of HIV-1 infected individuals [31]. Intriguingly, downregulation of miR-125b has been implicated in higher level of viremia in these patients. There is increasing evidence that cellular miRNAs may negatively regulate of HIV-1 by either targeting HIV-1 mRNAs, and/or modulate expression of cellular factors [32-33]. A survey of literature and bioinformatics analysis did not identify known cellular targets such as RANTES, Sigma-1 receptor, MIP-la/b, etc., that are known to play a role in cocaine induced enhancement of HIV-1 replication. Although we cannot exclude the indirect effects of miR-125b, it is likely that the observed effects of cocaine on HIV-1 replication is most likely due to direct targeting of HIV-1 genome by miR-125b. Therefore, our findings allude to novel mechanisms/pathways by which cocaine may enhance HIV-1 replication in CD4+ $\mathrm{T}$ cells. Most importantly, identifying the targets of miR-125b in CD4+ T cells will help us decipher the mechanisms/pathways targeted by cocaine. This knowledge will culminate in understanding the basis for increased HIV-1 pathogenesis among cocaine addicts and identifying potentially novel targets for future therapeutic intervention.

\section{Conclusions}

This study demonstrates for the first time that cocaine enhances HIV-1 replication in primary CD4+ T cells by downregulating the antiviral innate immunity of cellular miRNA "miR-125b". Since the accepted paradigm underlying cocaine-induced enhancement of HIV-1 replication is focused on viral entry, data presented here adds to the complexity of interplay between cocaine and HIV/ AIDS by revealing involvement of HIV-1 post entry steps.

\section{Author Contributions}

Conceived and designed the experiments: $\mathrm{CD}$ designed and directed the entire study. Performed the experiments: CM, JM and JP carried out the real-time PCR, HIV-1 infection, and knockdown experiments. Analyzed the data: CM, JP and CD analyzed the data. Contributed reagents/ materials/analysis tools: CM, JM and JP carried out the real-time PCR, HIV-1 infection, and knockdown experiments. Wrote the manuscript: CM, $\mathrm{JP}$ and $\mathrm{CD}$ wrote the manuscript and all the authors reviewed the manuscript.

6. Khalsa JH, Royal W (2004) Do drugs of abuse impact on HIV disease. J. Neuroimmunol. 147: 6-8.

7. Friedman H, Pross S, Klein TW (2006) Addictive drugs and their relationship with infectious diseases. FEMS Immunol Med Microbiol, 47: 330-342.

8. Kopnisky KL, Bao J, Lin YW (2007) Neurobiology of HIV, psychiatric and substance abuse comorbidity research: workshop report. Brain Behav Immun 21: 428-441.

9. Chaisson RW, Bacchetti P, Osmond D, Brodie B, Sande MA, et al. (1989) Cocaine use and HIV infection in intravenous drug users in San Francisco. JAMA 261: 561-565. 
10. Anthony JC, Vlahov D, Nelson KE, Cohn S, Astemborski J, et al. (1991) New evidence on intravenous cocaine use and the risk of infection with human immunodeficiency virus type 1. Am J Epidemiol 134: 1175-1189.

11. Baum MK, Rafie C, Lai S, Sales S, Page B, et al. (2009) Crack-Cocaine Use Accelerates HIV Disease Progression in a Cohort of HIV-Positive Drug Users. J Acquir Immune Defic Syndr 50: 93-99.

12. Duncan R, Shapshak P, Page JB, Chiappelli F, McCoy CB, et al. (2007) Crack cocaine: effect modifier of RNA viral load and CD4 count in HIV infected African American women. Front Biosci 12: 488-495.

13. Cook JA, Burke-Miller JK, Cohen MH, Cook RL, Vlahov D, et al. (2008) Crack cocaine, disease progression, and mortality in a multicenter cohort of HIV-1 positive women. AIDS 22: 1355-1363.

14. Peterson PK, Gekker G, Chao CG, Schut R, Molitor TW, et al. (1991) Cocaine potentiates HIV-1 replication in human peripheral blood mononuclear cell cocultures. Involvement of transforming growth factor-beta. J Immunol 146: 8184.

15. Bagasra O, Pomerantz RJ (1993) Human immunodeficiency virus type 1 replication in peripheral blood mononuclear cells in the presence of cocaine. J Infect Dis 168: 1157-1164.

16. Roth MD, Tashkin DP, Choi R, Jamieson BD, Zack JA, et al. (2002) Cocaine enhances human immunodeficiency virus replication in a model of severe combined immunodeficient mice implanted with human peripheral blood leukocytes. J Infect Dis 2002 185: 701-705.

17. Rofael HZ, Turkall RM, Abdel-Rahman MS (2003) Immunomodulation by cocaine and ketamine in postnatal rats. Toxicology 188: 101-14.

18. Watzl B, Watson RR (1990) Immunomodulation by cocaine-a neuroendocrine mediated response. Life Sci 46: 1319-29.

19. Halpern JH, Sholar MB, Glowacki J, Mello NK, Mendelson JH, et al. (2003) Diminished interleukin-6 response to proinflammatory challenge in men and women after intravenous cocaine administration. J Clin Endocrinol Metab 88(3): 1188-1193.

20. Nair MP, Mahajan S, Chadha KC, Nair NM, Hewitt RG, et al. (2001) Effect of cocaine on chemokine and CCR-5 gene expression by mononuclear cells from normal donors and HIV-1 infected patients. Adv Exp Med Biol 493: 235-40.

21. Nair MP, Chadha KC, Hewitt RG, Mahajan S, Sweet A, et al. (2000) Cocaine differentially modulates chemokine production by mononuclear cells from normal donors and human immunodeficiency virus type 1-infected patients. Clin Diagn Lab Immunol 7(1): 96-100.

22. Cocchi F, DeVico AL, Garzino-Demo A, Arya SK, Gallo SK et al. (1995) Identification of RANTES, MIP-1a MIP-1b as the major HIV suppressive factors produced by CD81 T cells. Science 270: 1811-1815.

23. Reynolds JL, Mahajan SD, Aalinkee IR, Nair B, Sykes DE, et al. (2009) Modulation of the Proteome of Peripheral Blood Mononuclear Cells from HIV1-Infected Patients by Drugs of Abuse. J Clin Immunol 29: 646-656.

24. Telesnitsky A, Goff SP (1997) Reverse Transcriptase and the Generation of Retroviral DNA: In Coffin JM, Hughes SH, Varmus HEeditors. Retroviruses. Cold Spring Harbor Laboratory Press, Plainview, New York, 121-160.

25. Bartel DP (2004) MicroRNAs: Genomics, Biogenesis, Mechanism, and Function. Cell 116(2): 281-297.

26. Shaham L, Binder V, Gefen N, Borkhardt A, Izraeli S (2012): MiR-125 in normal and malignant hematopoiesis. Leukemia. doi: 10.1038/leu.2012.90. [Epub ahead of print].
27. Lagos-Quintana M, Rauhut R, Yalcin A, Meyer J, Lendeckel W, et al. (2002) Curr Biol 12: 735-739.

28. Rossi RL, Rossetti G, Wenandy L, Curti S, Ripamonti A, et al. (2011) Distinct microRNA signatures in human lymphocyte subsets and enforcement of the naive state in CD4(+) T cells by the microRNA miR-125b. Nat Immunol 12(8): 796-803.

29. Huang J, Wang F, Argyris E, Chen K, Liang Z, et al. (2007) Cellular microRNAs contribute to HIV-1 latency in resting primary CD4+ T lymphocytes. Nat Med 13: 1241-1247.

30. Wang X, Ye L, Hou W, Zhou Y, Wang YJ, et al. (2009) Cellular microRNA expression correlates with susceptibility of monocytes/macrophages to HIV-1 infection. Blood 113: 671-674.

31. Witwer KW, Watson AK, Blankson JN, Clements JE (2012) Relationships of PBMC microRNA expression, plasma viral load, and CD4+ T-cell count in HIV-1-infected elite suppressors and viremic patients. Retrovirology 9: 5 .

32. Chiang K, Rice AP (2011): Mini ways to stop a virus: microRNAs and HIV-1 replication Future Virol 6: 209-221.

33. Hariharan M, Scaria V, Pillai B, Brahmachari SK (2005) Targets for human encoded microRNAs in HIV genes. Biochem Biophys Res Commun 337: 1214 1218 .

34. Clouse KA, Powell D, Washington I, Poli G, Strebel K, et al. (1989) Monokine regulation of human immunodeficiency virus-1 expression in a chronically infected human T cell clone. J Immunol 142: 431-438.

35. Folks TM, Glouse KA, Justement J, Rabson A, Duh E, et al. (1989). Tumor necrosis factor a induces expression of human immunodeficiency virus in a chronically infected T-cell clone. Proc Natl Acad Sci U S A 86: 2365-2368.

36. Wei X, Decker JM, Liu H, Zhang Z, Arani RB, et al. (2002) Emergence of resistant human immunodeficiency virus type 1 in patients receiving fusion inhibitor (T-20) monotherapy. Antimicrob Agents Chemother 46: 1896-1905.

37. Stephens BG, Jentzen JM, Karch S, Mash DC, Wetli CV (2004) Criteria for the interpretation of cocaine levels in human biological samples and their relation to the cause of death. Am J Forensic Med Pathol 25: 1-10.

38. Van Dyke C, Barash PG, Jatlow P, Byck R (1976) Cocaine: plasma concentrations after intranasal application in man. Science 191: 859-861.

39. Broder CC, Collman RG (1997) Chemokine receptors and HIV. J Leukoc Biol 62: 20-29.

40. Chan D, Kim P (1998) HIV entry and its inhibition. Cell 93: 681-684.

41. Wyatt R, Sodroski J (1998) The HIV-1 envelope glycoproteins: fusogens, antigens, and immunogens. Science 280: 1884-1888.

42. Aiken C (1997) Pseudotyping human immunodeficiency virus type 1 (HIV-1) by the glycoprotein of vesicular stomatitis virus targets HIV-1 entry to an endocytic pathway and suppresses both the requirement for Nef and the sensitivity to cyclosporin A. J Virol 71: 5871-5877.

43. Morey JS, Ryan JC, Van Dolah FM (2006). Microarray validation: factors influencing correlation between oligonucleotide microarrays and real-time PCR. Biol Proced Online 8: 175-193.

44. Wang X, Ye L, Zhou Y, Liu MQ Zhou DJ, Ho WZ (2011) Inhibition of antiHIV microRNA expression: a mechanism for opioid-mediated enhancement of HIV infection of monocytes. Am J Pathol. 178: 41-47. 